\title{
Changes in volume of various retinal layers over time in early and intermediate age-related macular degeneration
}

\author{
Ali Lamin ${ }^{1,2} \cdot$ Jonathan D. Oakley ${ }^{3} \cdot$ Adam M. Dubis $^{1,2} \cdot$ Daniel B. Russakoff ${ }^{3} \cdot$ Sobha Sivaprasad $\oplus^{1,2}$
}

Received: 15 July 2018 / Accepted: 24 September 2018 / Published online: 11 October 2018

(c) The Royal College of Ophthalmologists 2018

\begin{abstract}
Purpose To evaluate longitudinally volume changes in inner and outer retinal layers in early and intermediate age-related macular degeneration (AMD) compared to healthy control eyes using optical coherence tomography (OCT).

Methods 71 eyes with AMD and 31 control eyes were imaged at two time points: baseline and after 2 years. Automated OCT layer segmentation was performed using Orion $^{\mathrm{TM}}$. This software is able to measure volumes of retinal layers with distinct boundaries including Retinal Nerve Fibre Layer (RNFL), Ganglion Cell-Inner Plexiform Layer (GCIPL), Inner Nuclear Layer (INL), Outer Plexiform Layer (OPL), Outer Nuclear Layer (ONL), Photoreceptors (PR) and Retinal Pigment Epithelium-Bruch's Membrane complex (RPE-BM). The mean retinal layer volumes and volume changes at 2 years were compared between groups.

Results Mean GCIPL and INL volumes were lower, while PR and RPE-BM volumes were higher in AMD eyes than controls at baseline (all $P<0.05$ ) and year 2 (all $P<0.05$ ). In AMD eyes, RNFL and ONL volumes decreased by 0.0232 $(P=0.033)$ and $0.0851(P=0.001)$, respectively. In contrast, OPL and RPE-BM volumes increased in AMD eyes by $0.0391(P=0.000)$ and $0.0209(P=0.000)$ respectively. Moreover, there were significant differences in longitudinal volume change of OPL $(P=0.02)$, ONL $(P=0.008)$ and RPE-BM $(P=0.02)$ between AMD eyes and controls.

Conclusions There were abnormal retinal layer volumes and volume changes in eyes with early and intermediate AMD.
\end{abstract}

\section{Introduction}

Age-related macular degeneration (AMD) is one of the major causes of irreversible vision loss and blindness among people over 50 years of age in the western world [1-3]. It is a progressive degenerative disease of the macula and is generally considered as a disease of the outer retina. AMD can be classified clinically into early, intermediate and advanced stage to characterise disease severity [4]. There are two advanced forms of AMD: neovascular (exudative or wet) AMD (nAMD) and geographic atrophy (GA).

Sobha Sivaprasad

sobha.sivaprasad@moorfields.nhs.uk

1 NIHR Moorfields Biomedical Research Centre, Moorfields Eye Hospital NHS Foundation Trust, London, UK

2 UCL Institute of Ophthalmology, London, UK

3 Voxeleron LLC, Pleasanton, CA, USA
Although nAMD is less common than GA, nAMD is responsible for $90 \%$ of all advanced cases of severe visual loss and legal blindness due to the development of choroidal neovascularisation (CNV) [5]. CNV is abnormal choroidal blood vessels that grow through Bruch membrane into the subretinal space, leading to the accumulation of subretinal fluid and pigment epithelium detachment [6, 7]. CNV can develop into a discform scar as it matures. Therefore, there is an unmet need to identify biomarkers that can fully predict the progression to nAMD in order to allow early intervention before permanent damage.

Different retinal layers were described histologically as being affected by AMD. Photoreceptor cells loss was found to occur in the earlier stage of AMD [8-10], while an atrophy of the ganglion cell layer (GCL) occurs in the late stage of the disease [10].

The advancement in OCT's image resolution have allowed us to compare in vivo retinal layers to the histological structure and improve segmentation to assess individual retinal layers. Several studies performed manual segmentation of retina and demonstrated that there are 
changes in the photoreceptor layer thickness, which is affected by drusen dynamics [11-14].

Recently, automated segmentation software programmes that enable analysis layers of the retina qualitatively and quantitatively have been introduced $[15,16]$. Using these softwares, recent studies of AMD eyes have demonstrated that there were a change not only in the outer retinal layers as previously established but also in the inner retina such as the ganglion cell complex (GCC) thickness, which includes GCL and inner plexiform layer (IPL) [17-21].

The aim of this study is to look at change in retinal layer volumetric measures on OCT in eyes with early/intermediate AMD compared to a control population over time to understand the longitudinal changes in these layers.

\section{Methods}

\section{Participants}

This retrospective, observational, longitudinal study was approved by the Ethical Review Board of Moorfields Eye Hospital (ROAD 17/004) and adhered to the principles of the Declaration of Helsinki. The study was conducted between 2017 and 2018 at Moorfields Eye Hospital, London, UK.

To be included in the study, subject eyes had to have been imaged with spectral domain (SD) OCT (Topcon 3D1000) at two time points (baseline and year 2). The inclusion criteria for the AMD eyes included early or intermediate AMD in the study eye and neovascualr AMD in the fellow eye. Study eyes with the following criteria were excluded from the study: poor quality images (signal strength $<30$ ) and/or advanced AMD including GA or $\mathrm{CNV}$, high refractive error ( $\geq 6$ dioptres), glaucoma, diabetic retinopathy, previous retinal surgery or any other posterior segment disease.

A control group of healthy individuals was also included in the study. A baseline demographic data including age, sex and laterality was recorded for all participants.

\section{OCT imaging}

Each eye macular area within $6 \times 6 \mathrm{~mm}^{2}$ macular cube centred on fovea was scanned two times over 2 years with Topcon 3D-1000 using $512 \times 128$ scan pattern.

\section{OCT segmentation}

OCT segmentation was performed using Orion $^{\mathrm{TM}}$ (Voxeleron, Pleasanton, CA) that can perform automated separation of the retina into individual layers and measure their volumes in the central macular area $(6 \mathrm{~mm}$ diameter $)$ automatically centred on the fovea by the software, thereby

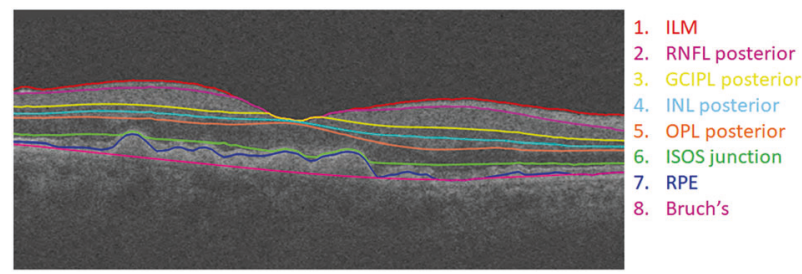

Fig. 1 Retinal layer segmentation with detectable layer boundaries in an AMD eye as analysed automatically by the Orion software. Layers $1-2=$ Retinal Nerve Fibre Layer (RNFL); Layers 2-3= Ganglion Cell Ganglion Cell and Inner Plexiform Layer (GCIPL); Layers 3-4 = Inner Nuclear Layer (INL); Layers 4-5= Outer Plexiform Layer (OPL); Layers 5-6 = Outer Nuclear Layer (ONL); Layers 6-7 = Photoreceptor complex (PR); Layers 7-8= Retinal Pigment Epithelium-Bruch's Membrane complex (RPE-BM); Layers 1-8= Total Retinal Layers

supporting longitudinal analysis. The retina was segmented into the following 7 layers (Fig. 1): (1) Retinal Nerve Fibre Layer (RNFL), (2) Ganglion Cell and Inner Plexiform Layer (GCIPL), (3) Inner Nuclear Layer (INL), (4) Outer Plexiform Layer (OPL), (5) Outer Nuclear Layer (ONL), (6) Photoreceptors (PR) and (7) Retinal Pigment EpitheliumBruch's Membrane complex (RPE-BM).

\section{Statistics}

Comparisons of mean of all retinal layer volumes between AMD eyes and control eyes were performed at baseline and year 2. Volume changes from baseline to year 2 were also compared in each group and between the 2 groups. A test for normality (Shapiro-Wilks) was performed for each parameter. Where the distribution found to be normal, Student's $t$-test was used; otherwise, nonparametric $t$-tests (Mann-Whitney U and Wilcoxon signed-rank) were used. All statistical analyses were performed using SPSS software (IBM SPSS Statistics for Windows, Version 24.0). $P$ values of $<0.05$ were considered statistically significant.

\section{Results}

\section{Demographics of participants}

102 eyes of 102 individuals were included in the study. 71 participants (42 females [59.2\%] and 28 males [39.4\%]) were included in the AMD group and 31 participants (19 females [61.3\%] and 12 males [38.7\%]) were included in the control group. Mean (SD) ages were 74 (8.5) years and 64.1 (6.4) years for AMD and controls, respectively.

\section{Mean retinal layer volumes at baseline and year 2}

Table 1 shows mean volumes of individual retinal layers in the central macular area $(6 \mathrm{~mm}$ diameter $)$ measured at two 
Table 1 Mean Retinal Layer Volumes at Baseline and Year 2 in Age-Related Macular Degeneration Eyes $(\mathrm{n}=71)$ and Control Eyes $(\mathrm{n}=31)$

\begin{tabular}{|c|c|c|c|c|}
\hline \multicolumn{2}{|c|}{ Retinal Layer and Time Point } & \multirow{2}{*}{$\begin{array}{l}\text { AMD Group }(\mathrm{n}=71) \\
\text { Mean (SD) } \\
8.22(.44)\end{array}$} & \multirow{2}{*}{$\begin{array}{l}\text { Control } \\
\text { Group } \\
(\mathrm{n}=31) \\
\text { Mean (SD) }\end{array}$} & \multirow{2}{*}{$\begin{array}{l}P \\
\text { value } \\
0.469\end{array}$} \\
\hline TRV & Baseline & & & \\
\hline & Year 2 & $8.16(.45)$ & $8.23(.42)$ & 0.563 \\
\hline \multirow[t]{2}{*}{ RNFL } & Baseline & $1.12(.13)$ & $1.09(.08)$ & 0.506 \\
\hline & Year 2 & $1.09(.13)$ & $1.08(.07)$ & 0.438 \\
\hline \multirow[t]{2}{*}{ GCIPL } & Baseline & $1.84(.19)$ & $1.97(.14)$ & 0.001 \\
\hline & Year 2 & $1.85(.18)$ & $1.95(.16)$ & 0.011 \\
\hline \multirow[t]{2}{*}{ INL } & Baseline & $1.00(.07)$ & $1.03(.06)$ & 0.026 \\
\hline & Year 2 & $.990(.07)$ & $1.03(.06)$ & 0.014 \\
\hline \multirow[t]{2}{*}{ OPL } & Baseline & $.666(.12)$ & $.672(.06)$ & 0.544 \\
\hline & Year 2 & $.705(.11)$ & $.675(.07)$ & 0.133 \\
\hline \multirow[t]{2}{*}{ ONL } & Baseline & $2.29(.22)$ & $2.24(.17)$ & 0.438 \\
\hline & Year 2 & $2.21(.21)$ & $2.23(.16)$ & 0.426 \\
\hline \multirow[t]{2}{*}{ PR } & Baseline & $1.28(.09)$ & $1.25(.06)$ & 0.006 \\
\hline & Year 2 & $1.30(.08)$ & $1.25(.06)$ & 0.008 \\
\hline \multirow[t]{2}{*}{ RPE-BM } & Baseline & $.148(.08)$ & $.102(.11)$ & 0.000 \\
\hline & Year 2 & $.169(.11)$ & $.102(.09)$ & 0.000 \\
\hline
\end{tabular}

Statistically significant values with $P<0.05$ are in bold.

$T R V=$ Total Retinal Volume; $R N F L=$ Retinal Nerve fiber Layer; $G C I P L=$ Ganglion Cell and Inner Plexiform Layer; $I N L=$ Inner Nuclear Layer; $O P L=$ Outer Plexiform Layer; $O N L=$ Outer Nuclear Layer; $P R=$ Photoreceptors; $R P E-B M=$ Retinal Pigment-Epithelium-Bruch's Membrane complex time points, baseline and year 2 . There were statistically significant differences between the 2 groups in mean volumes of GCIPL, INL, PR and RPE-BM at both baseline and year 2. GCIPL and INL volumes were smaller in AMD eyes than controls at baseline (both $P<0.05$ ) and year 2 (both $P<0.05$ ). Conversely, PR and RPE-BM volumes were greater in AMD eyes than controls at baseline (both $P<0.05$ ) and year 2 (both $P<0.05$ ). Otherwise, no significant deference was found between the two groups in volumes of other retinal layers.

\section{Longitudinal volume change from baseline to year 2}

Longitudinal comparisons from baseline to year 2 in AMD and control eyes are shown in Table 2. Mean total retinal volume (TRV) decreased significantly from baseline to year 2 in both AMD and control eyes by $0.0561(P=0.018)$ and $0.0579(P=0.011)$ respectively. From baseline to year 2 in AMD eyes, mean volumes of RNFL and ONL decreased by $0.0232(P=0.033)$ and $0.0851(P=0.001)$ respectively. In contrast, there were significant increase in mean volumes of OPL and RPE-BM in AMD eyes by $0.0391(P=0.000)$ and $0.0209(P=0.000)$, respectively.

From baseline to year 2, there were significant differences between AMD eyes and controls in longitudinal volume change of OPL $(P=0.02)$, ONL $(P=0.008)$ and RPE-BM $\quad(P=0.02)$. Otherwise, no significant deference was found between the 2 groups in the longitudinal volume change from baseline to year 2 of other retinal layers.

Differentiation between AMD and control groups by volume measurements and longitudinal volume change are shown in Fig. 2. It can be noted that, despite some overlap existed between AMD and controls, the GCIPL, INL and ONL volumes of the AMD eyes were reduced substantially compared to control eyes.

\section{Conclusions}

The present study showed that some retinal layer volumes are significantly different between AMD and control eyes. The volume of inner retinal layers (GCIPL and INL) were lesser in AMD eyes than in controls, while the volume of outer retinal layers (PR and RPE-BM) were greater in AMD eyes as compared to controls. In addition, some retinal layers underwent volume change in AMD eyes over the 2 -year follow-up. There was a reduction in ONL volume in AMD eyes over the follow-up period. In contrast, OPL and RPE-BM increased in volume from baseline to year 2 in 
Table 2 Longitudinal Change in Retinal Layer Volumes in Age-Related Macular Degeneration Eyes $(\mathrm{n}=71)$ and Control Eyes $(\mathrm{n}=31)$

\begin{tabular}{|c|c|c|c|c|}
\hline \multicolumn{2}{|c|}{$\begin{array}{l}\text { Retinal Layer and } \\
\text { Group }\end{array}$} & \multirow{2}{*}{$\begin{array}{l}\text { Volume Change } \\
\text { (Baseline - Year 2) } \\
\text { Mean (SD) } \\
\mathbf{0 5 6 1}(\mathbf{. 1 9 5 )}\end{array}$} & \multirow{2}{*}{$\begin{array}{l}P \text { value }^{\mathrm{a}} \\
\mathbf{0 . 0 1 8}\end{array}$} & \multirow{2}{*}{$\frac{P \text { value }^{\mathrm{b}}}{0.956}$} \\
\hline TRV & AMD & & & \\
\hline & Control & .0579 (.118) & 0.011 & \\
\hline \multirow[t]{2}{*}{ RNFL } & AMD & $.0232(.09)$ & 0.033 & 0.904 \\
\hline & Control & $.0184(.05)$ & 0.052 & \\
\hline \multirow[t]{2}{*}{ GCIPL } & AMD & $-.0077(.114)$ & 0.324 & 0.757 \\
\hline & Control & $.0171(.074)$ & 0.075 & \\
\hline \multirow[t]{2}{*}{ INL } & AMD & $.0131(.056)$ & 0.053 & 0.361 \\
\hline & Control & $.0035(.045)$ & 0.672 & \\
\hline \multirow[t]{2}{*}{ OPL } & AMD & $-.0391(.094)$ & 0.000 & 0.02 \\
\hline & Control & $-.0028(.058)$ & 0.792 & \\
\hline \multirow[t]{2}{*}{ ONL } & AMD & .0851 (.148) & 0.001 & 0.008 \\
\hline & Control & $.0206(.06)$ & 0.799 & \\
\hline \multirow[t]{2}{*}{ PR } & AMD & $-.0184(.072)$ & 0.130 & 0.175 \\
\hline & Control & $.0011(.067)$ & 0.926 & \\
\hline \multirow[t]{2}{*}{ RPE-BM } & AMD & $-.0209(.052)$ & 0.000 & 0.02 \\
\hline & Control & $.0007(.023)$ & 0.754 & \\
\hline
\end{tabular}

Statistically significant values with $P<0.05$ are in bold.

$T R V=$ Total Retinal Volume; $R N F L=$ Retinal Nerve Fiber Layer; $G C I P L=$ Ganglion Cell and Inner Plexiform Layer; $I N L=$ Inner Nuclear Layer; $O P L=$ Outer Plexiform Layer; $O N L=$ Outer Nuclear Layer; $P R=$ Photoreceptors; $R P E-B M=$ Retinal Pigment EpitheliumBruch's Membrane complex

${ }^{a}$ Baseline versus Year 2

${ }^{\mathrm{b}} \mathrm{AMD}$ group versus Control group

AMD eyes. These findings suggest that GCIPL and INL undergo thinning earlier than ONL in the disease process.

The current finding of GCIPL and INL thinning in early and intermediate AMD is consistent with previous studies using OCT [17-23]. Different theories were proposed to explain the underling mechanism of GCIPL and INL thinning. One theory is that this may occur as a result of superficial retinal microvascular abnormality that leads to reduction in inner retina perfusion and ischaemia [24-27]. However, the exact reason for superficial retinal vascular changes in AMD remains unclear. Another theory is that synaptic malformation due to photoreceptors loss could lead to reducing transneuronal input to inner retina, triggering the inner retina degeneration process [28].

The expansion in OPL layer volume in AMD eyes during the 2-year follow-up should be interpreted with caution as this layer is difficult to image using OCT because a change in the angle of incidence of the OCT beam on the OPL can show it as thicker if Henle's fibres are revealed. Being a thin, plexiform layer, its variance is therefore high. So if this change is indeed real, it could occur mechanically as the retina attempts to preserve structural integrity through the role of the Muller cells. It is also consistent with a light microscopic study that demonstrated that there is a displacement of nuclei from the ONL into the OPL secondary to shrinkage of their attached fibres [29].

Despite no statistically difference in ONL volume between AMD eyes and controls, the reduction in ONL volume from baseline to year 2 in AMD eyes was statistically significant and this may indicate later degeneration of the layer or a secondary RPE disruption caused by the increasing drusen material. This finding is consistent with histopathology result that demonstrated ONL thinning in ageing models [29-31]. These findings have also been confirmed by recent studies using OCT that showed ONL thinning in eyes with intermediate AMD $[11,13]$.

Unexpected was the finding of expanded PR in AMD eyes which could be due to swelling of the cone distal axon as shown in a histological study [31]. Similar to OPL expansion, PR expansion might be due to displacement of nuclei from the ONL to the layer of rods and cones [29]. Behbehani et al. also observed this in patients with multiple sclerosis and concluded that this was not "compensatory" thickening to the GCIPL thinning [32].

Another finding of the current study is that RPE-BM volume was greater in AMD eyes than controls and there was a significant increase in its volume from baseline to year 2 . These findings can be explained in term of drusen formation, extracellular deposits that accumulate between RPE and BM. A previous study found that RPE-BM thickening was associated with progression to advanced AMD [33].

This is, to our knowledge, the first study to explore longitudinally the change in retinal layer volumes in AMD eyes using OCT. Moreover, our study evaluated both the inner and outer retina that was not done by most of the previous studies.

In summary, our data showed abnormal retinal layer volumes and volume changes in eyes with early and intermediate AMD. The mean GCIPL and INL volumes were lower in eyes with AMD as compared to control eyes, while the mean PR and RPE-BM volumes were higher. Moreover, there were significant change in ONL (reduction), OPL (expansion) and RPE-BM (expansion) volumes during the 2-year follow-up in AMD eyes. These findings suggest that GCIPL and INL damage may precede ONL damage in eyes with AMD and migration of the latter nuclei to adjacent layers (OPL and RP) may be the cause of their expansions.

\section{Summary}

\section{What was known before}

- Although AMD is an outer retinal disease, inner retina may be affected as shown in previous cross sectional studies. 
Fig. 2 Box plots showing longitudinal volume change of total retinal volume (TRV),

Retinal Nerve Fibre Layer

(RNFL), Ganglion Cell

Ganglion Cell and Inner

Plexiform Layer (GCIPL), Inner

Nuclear Layer (INL), Outer

Plexiform Layer (OPL), Outer

Nuclear Layer (ONL),

Photoreceptors (PR) and Retinal

Pigment Epithelium-Bruch's

Membrane complex (RPE-BM).

AMD age-related macular degeneration
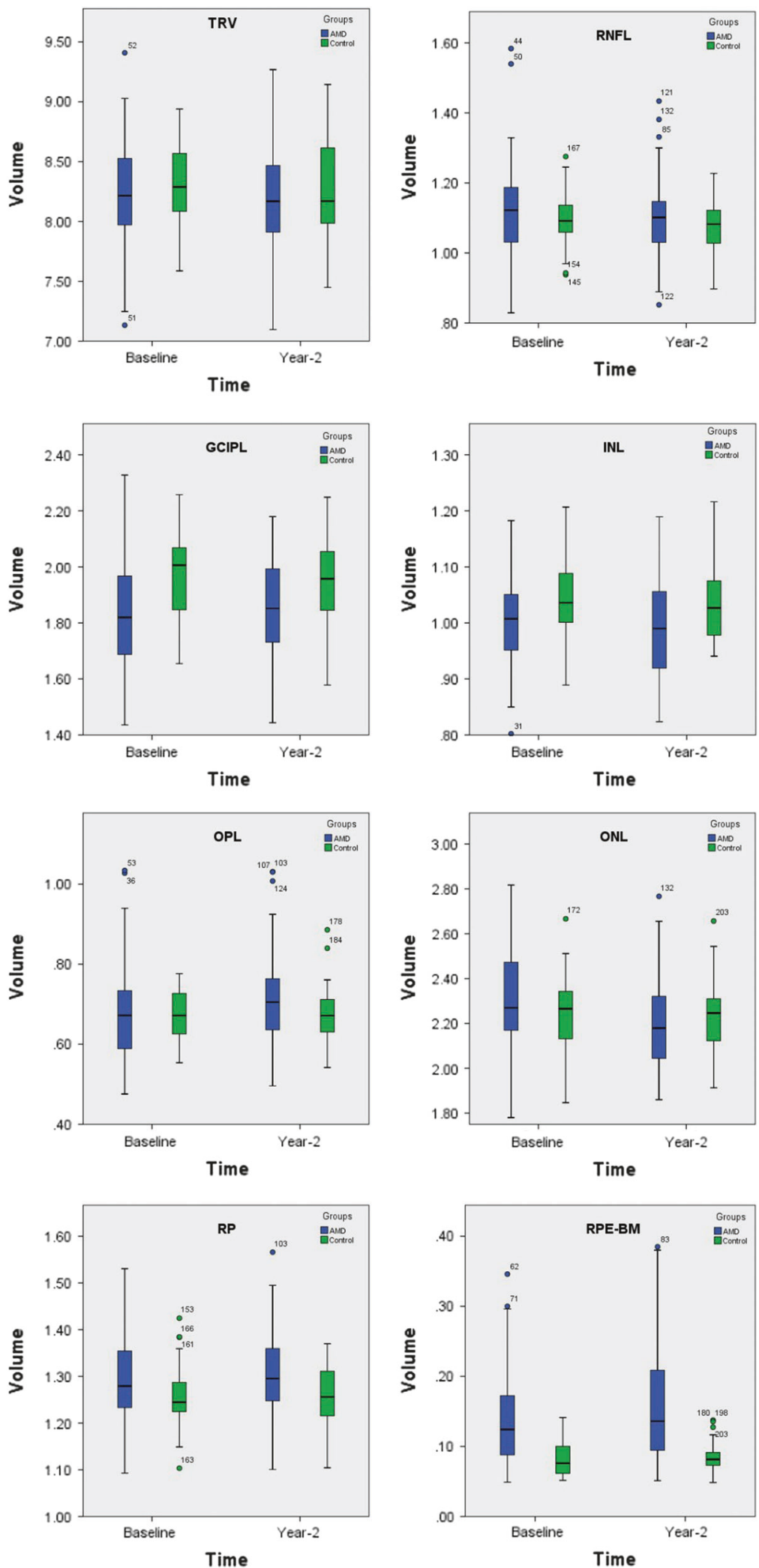


\section{What this study adds}

- Our study shows that both inner and outer retinal layers volume measurements change over time in eyes with early and intermediate AMD.

Acknowledgements The research was supported by the National Institute for Health Research (NIHR) Biomedical Research Centre based at Moorfields Eye Hospital NHS Foundation Trust and UCL Institute of Ophthalmology. The views expressed are those of the author(s) and not necessarily those of the NHS, the NIHR or the Department of Health.

\section{Compliance with ethical standards}

Conflict of interest The authors declare that they have no conflict of interest.

\section{References}

1. van Leeuwen R, Klaver CC, Vingerling JR, Hofman A, de Jong PT. Epidemiology of age-related maculopathy: a review. Eur $\mathbf{J}$ Epidemiol. 2003;18:845-54.

2. Friedman DS, O’Colmain BJ, Munoz B, Tomany SC, McCarty C, de Jong PT, et al. Prevalence of age-related macular degeneration in the United States. Arch Ophthalmol. 2004;122:564-72.

3. Resnikoff S, Pascolini D, Etya'ale D, Kocur I, Pararajasegaram R, Pokharel GP, et al. Global data on visual impairment in the year 2002. Bull World Health Organ. 2004;82:844-51.

4. Ferris FL 3rd, Wilkinson CP, Bird A, Chakravarthy U, Chew E, Csaky K, et al. Clinical classification of age-related macular degeneration. Ophthalmology. 2013;120:844-51.

5. Ferris FL 3rd, Fine SL, Hyman L. Age-related macular degeneration and blindness due to neovascular maculopathy. Arch Ophthalmol. 1984;102:1640-2.

6. Green WR, Enger C. Age-related macular degeneration histopathologic studies. The 1992 Lorenz E. Zimmerman Lecture. Ophthalmology. 1993;100:1519-35.

7. Campochiaro PA, Soloway P, Ryan SJ, Miller JW. The pathogenesis of choroidal neovascularization in patients with agerelated macular degeneration. Mol Vis. 1999;5:34.

8. Curcio CA, Medeiros NE, Millican CL. Photoreceptor loss in agerelated macular degeneration. Invest Ophthalmol Vis Sci. 1996;37:1236-49.

9. Jackson GR, Owsley C, Curcio CA. Photoreceptor degeneration and dysfunction in aging and age-related maculopathy. Ageing Res Rev. 2002;1:381-96.

10. Medeiros NE, Curcio CA. Preservation of ganglion cell layer neurons in age-related macular degeneration. Invest Ophthalmol Vis Sci. 2001;42:795-803.

11. Schuman SG, Koreishi AF, Farsiu S, Jung SH, Izatt JA, Toth CA. Photoreceptor layer thinning over drusen in eyes with age-related macular degeneration imaged in vivo with spectral-domain optical coherence tomography. Ophthalmology. 2009;116: 488-96.e2.

12. Hartmann KI, Gomez ML, Bartsch DU, Schuster AK, Freeman WR. Effect of change in drusen evolution on photoreceptor inner segment/outer segment junction. Retina. 2012;32:1492-9.
13. Sadigh S, Cideciyan AV, Sumaroka A, Huang WC, Luo X, Swider M, et al. Abnormal thickening as well as thinning of the photoreceptor layer in intermediate age-related macular degeneration. Invest Ophthalmol Vis Sci. 2013;54:1603-12.

14. Sadigh S, Luo X, Cideciyan AV, Sumaroka A, Boxley SL, Hall LM, et al. Drusen and photoreceptor abnormalities in AfricanAmericans with intermediate non-neovascular age-related macular degeneration. Curr Eye Res. 2015;40:398-406.

15. Tan J, Yang Y, Jiang H, Liu C, Deng Z, Lam BL, et al. The measurement repeatability using different partition methods of intraretinal tomographic thickness maps in healthy human subjects. Clin Ophthalmol. 2016;10:2403-15.

16. Terry L, Cassels N, Lu K, Acton JH, Margrain TH, North RV, et al. Automated retinal layer segmentation using spectral domain optical coherence tomography: evaluation of inter-session repeatability and agreement between devices. PLoS ONE. 2016;11:e0162001.

17. Savastano MC, Minnella AM, Tamburrino A, Giovinco G, Ventre S, Falsini B. Differential vulnerability of retinal layers to early age-related macular degeneration: evidence by SD-OCT segmentation analysis. Invest Ophthalmol Vis Sci. 2014;55:560-6.

18. Lee EK, Yu HG. Ganglion cell-inner plexiform layer and peripapillary retinal nerve fiber layer thicknesses in age-related macular degeneration. Invest Ophthalmol Vis Sci. 2015;56: 3976-83.

19. Yenice E, Sengun A, Soyugelen Demirok G, Turacli E. Ganglion cell complex thickness in nonexudative age-related macular degeneration. Eye. 2015;29:1076-80.

20. Zucchiatti I, Parodi MB, Pierro L, Cicinelli MV, Gagliardi M, Castellino $\mathrm{N}$, et al. Macular ganglion cell complex and retinal nerve fiber layer comparison in different stages of age-related macular degeneration. Am J Ophthalmol. 2015;160:602-7.e1.

21. Muftuoglu IK, Ramkumar HL, Bartsch DU, Meshi A, Gaber R, Freeman WR. Quantitative analysis of the inner retinal layer thicknesses in age-related macular degeneration using corrected optical coherence tomography segmentation. Retina. 2017;38: 1478-84.

22. Borrelli E, Abdelfattah NS, Uji A, Nittala MG, Boyer DS, Sadda SR. Postreceptor neuronal loss in intermediate age-related macular degeneration. Am J Ophthalmol. 2017;181:1-11.

23. Camacho P, Dutra-Medeiros M, Paris L. Ganglion cell complex in early and intermediate age-related macular degeneration: evidence by SD-OCT manual segmentation. Ophthalmologica. 2017;238: 31-43.

24. Villegas-Perez MP, Lawrence JM, Vidal-Sanz M, Lavail MM, Lund RD. Ganglion cell loss in RCS rat retina: a result of compression of axons by contracting intraretinal vessels linked to the pigment epithelium. J Comp Neurol. 1998;392:58-77.

25. Feigl B, Brown B, Lovie-Kitchin J, Swann P. Functional loss in early age-related maculopathy: the ischaemia postreceptoral hypothesis. Eye. 2007;21:689-96.

26. Toto L, Borrelli E, Di Antonio L, Carpineto P, Mastropasqua R. Retinal vascular plexuses' changes in dry age-related macular degeneration, evaluated by means of optical coherence tomography angiography. Retina. 2016;36:1566-72.

27. Toto L, Borrelli E, Mastropasqua R, Di Antonio L, Doronzo E, Carpineto $\mathrm{P}$, et al. Association between outer retinal alterations and microvascular changes in intermediate stage age-related macular degeneration: an optical coherence tomography angiography study. Br J Ophthalmol. 2017;101:774-9.

28. Strettoi E, Porciatti V, Falsini B, Pignatelli V, Rossi C. Morphological and functional abnormalities in the inner retina of the rd/rd mouse. J Neurosci: Off J Soc Neurosci. 2002;22: 5492-504. 
29. Gartner S, Henkind P. Aging and degeneration of the human macula. 1. Outer nuclear layer and photoreceptors. Br J Ophthalmol. 1981;65:23-8.

30. Machida S, Kondo M, Jamison JA, Khan NW, Kononen LT, Sugawara $\mathrm{T}$, et al. $\mathrm{P} 23 \mathrm{H}$ rhodopsin transgenic rat: correlation of retinal function with histopathology. Invest Ophthalmol Vis Sci. 2000;41:3200-9.

31. Shelley EJ, Madigan MC, Natoli R, Penfold PL, Provis JM. Cone degeneration in aging and age-related macular degeneration. Arch Ophthalmol. 2009;127:483-92.
32. Behbehani R, Abu Al-Hassan A, Al-Salahat A, Sriraman D, Oakley JD, Alroughani R. Optical coherence tomography segmentation analysis in relapsing remitting versus progressive multiple sclerosis. PLoS ONE. 2017;12: e0172120.

33. Ferrara D, Silver RE, Louzada RN, Novais EA, Collins GK, Seddon JM. Optical coherence tomography features preceding the onset of advanced age-related macular degeneration. Invest Ophthalmol Vis Sci. 2017;58:3519-29. 